\title{
One-way transmission through the subwavelength slit in nonsymmetric metallic gratings
}

\author{
Semih Cakmakyapan, ${ }^{1, *}$ Andriy E. Serebryannikov, ${ }^{2}$ Humeyra Caglayan, ${ }^{1}$ and Ekmel Ozbay ${ }^{1}$ \\ ${ }^{1}$ Nanotechnology Research Center, Department of Physics, Bilkent University, 06800 Ankara, Turkey \\ ${ }^{2}$ Technische Universitaet Hamburg-Harburg, D-21071 Hamburg, Germany \\ *Corresponding author: semihc@bilkent.edu.tr
}

Received March 11, 2010; revised June 16, 2010; accepted July 6, 2010;

posted July 13, 2010 (Doc. ID 125350); published July 27, 2010

\begin{abstract}
An approach for obtaining one-way transmission in the beaming regime is suggested that is based on the directional radiation of surface plasmons in nonsymmetric metallic gratings with a single slit. In contrast to the various nonsymmetric one-way diffraction gratings that have recently been proposed, the possibility of obtaining of narrow beams is demonstrated. Strong directional selectivity can appear a wide range of the observation angles, while the angle of incidence is retained. (c) 2010 Optical Society of America

OCIS codes: $\quad 050.2770,240.6690$.
\end{abstract}

Achieving optical isolation has been the focus of interest for a long time. Within the passive framework, it can be obtained using anisotropic [1,2] or nonlinear [3] materials. Recently, several grating structures have been proposed for freely propagating waves, which enable the realization of the regimes with strong directional selectivity, that are not the same but similar in some features to the conventional isolation [4-6]. They all only contain isotropic constituents, and therefore no transformation of the initially linear polarization of the incident wave occurs.

In particular, it has been shown for the branched slit metallic gratings with different periods of the front- and back-side interfaces (nonsymmetric gratings) at normal incidence, $\theta=0$, that higher-order transmittance can be either zero or nonzero, depending on whether the larger-period or smaller-period interface is illuminated [4]. In contrast to the slit gratings, in those based on the photonic crystals with nonisotropic dispersion, the zero order cannot be coupled to a Floquet-Bloch wave in the vicinity of $\theta=0$, so transmittance is zero in one direction and nonzero in the opposite direction, while $\theta$ is retained. Furthermore, a similar effect can be obtained at $\theta \neq 0$ in case of isotropic dispersion with the effective index of refraction as $0<n_{\text {eff }}<1$, for example, in the gratings containing low-permittivity layers []ㅡ, and in the photonic crystal gratings [5]. If only zero order is coupled to a wave propagating inside a grating, the interfaces are isolated from each other in the sense that the numbers of higher diffraction orders, which contribute to transmission and reflection, are determined only by the period of the corresponding interfaces. The common features of the approaches in [4-6] are that at least one higher order is propagating and that they operate with wide beams.

At the same time, beaming is known to be obtainable in metallic gratings with slits, owing to surface plasmons [7-11]. For example, a plane or corrugated interface between a Drude metal and dielectric medium can support surface plasmons [12]. Since the skin depth of the plane metallic surfaces in the microwave regime approaches zero, they cannot be supported. Pendry et al. theoretically showed that the metallic surfaces, which can be thought of as perfect electric conductors in microwave regime, can support surface-plasmon-like waves, known as designer surface plasmons, when the surface has subwavelength holes [13]. Later, it was demonstrated experimentally by Hibbins et al. that surface plasmons can appear on the metallic corrugated surfaces [14]. Coupling the light to the surface plasmons with a periodic structure on the input surface provides enhanced transmission through a subwavelength slit [15]. Generally, the transmitted wave is diffracted to every direction. However, if the output surface is designed with periodic grooves, beaming can appear $[\underline{7,8}]$. It was shown that changing the angle of propagation of the transmitted beam is possible in asymmetric gratings [9-11]. Basically, the grating structure of the input surface leads to enhanced transmission, while the grating structure on the output surface is responsible for beaming. In some sense, this feature is analogous to that observed in the one-way slit gratings, where the larger-period output interface is responsible only for the appearance of nonzero, higher-order transmittance [4]].

In this Letter, we will theoretically demonstrate that one-way transmission can be obtained in the beaming regime in nonsymmetric metallic gratings with a single subwavelength slit. This is achieved because of the radiating surface plasmons, which show different conditions of excitation for different grating periods of the input and output interfaces. Consideration is restricted here to the microwave frequencies. We designed two metallic grating structures with the centered subwavelength slit.

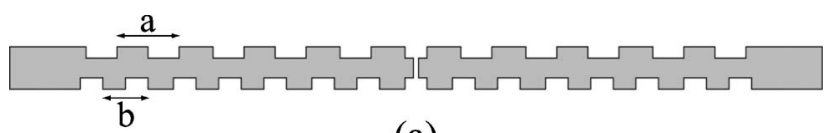

(a)

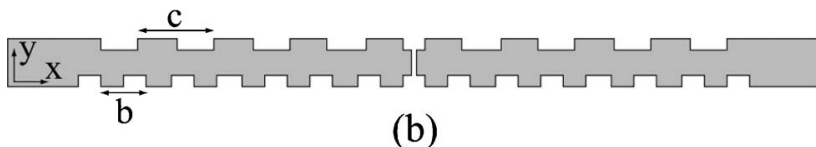

(b)

Fig. 1. Schematic of the metallic (Al) grating structures with subwavelength slit at the center, which is $2 \mathrm{~mm}$ wide and $8 \mathrm{~mm}$ long. (a) Sample 1: front-side surface period, $b=16 \mathrm{~mm}$; back-side surface period, $a=22 \mathrm{~mm}$. (b) Sample 2: front-side grating surface period, $b=16 \mathrm{~mm}$; back-side surface period, $c=26 \mathrm{~mm}$. 
They are schematically shown in Fig. 1 . Both the frontside and back-side interfaces have rectangular periodic grooves of a depth of $4 \mathrm{~mm}$. The obtained gratings are symmetric with respect to the slit axis and have different periods of the front- and back-side interfaces; see Fig. 1. The effect of changing the input (illumination) side has been studied at a fixed angle of incidence for both structures. Simulations were carried out by using an finitedifference time-domain technique.

Figure 2 shows transmission maps on the anglefrequency plane. They are calculated $30 \mathrm{~cm}$ away from the center of the structure. The front-side and back-side normal incidence illuminations of Sample 1 are presented in Figs. 2(a) and 2(b), respectively. The electric field is polarized here in the $x$ direction. The strongest transmission appears in both cases near $f=11.2 \mathrm{GHz}$. However, for the front-side illumination, transmission is significant only in the vicinity of the observation angle $\psi=0$. In contrast, for the back-side illumination, it is significant within a $\Psi$-range that is about $70 \mathrm{deg}$ wide. Therefore, this regime is similar to that occurring in the branched-slit and other one-way diffraction gratings [4-6]. Indeed, the transmission maxima at $\psi=0$ are analogous to the zero-order transmission, while the maxima near $\psi= \pm 18^{\circ}$ in Fig. 2(b) are analogous to the firstorder transmission in these gratings. However, in our case, the conditions of surface plasmon excitation are different for the two used illumination directions so that, strictly speaking, no reciprocal component in transmission should appear.

Transmission maps in Figs. 2(c) and 2(d) for Sample 2 are obtained by tilting the incident beam by $30^{\circ}$. It is shown that the range of significant transmission and, hence, the expected resonance frequencies are strongly distinguished, being dependent on the illumination side. For the front-side illumination of Sample 2, the resonance frequency $f \approx 9.8 \mathrm{GHz}$, while for the back-side illumination it is $f \approx 14.5 \mathrm{GHz}$. At the resonance frequency of one of the interfaces, the transmission for the other becomes close to zero. This is evidence for the effect of unidirectional transmission, which is similar to that observed in the photonic-crystal and ultralow-permittivity gratings [ㄷ,]. In our case, this effect appears in the beam-
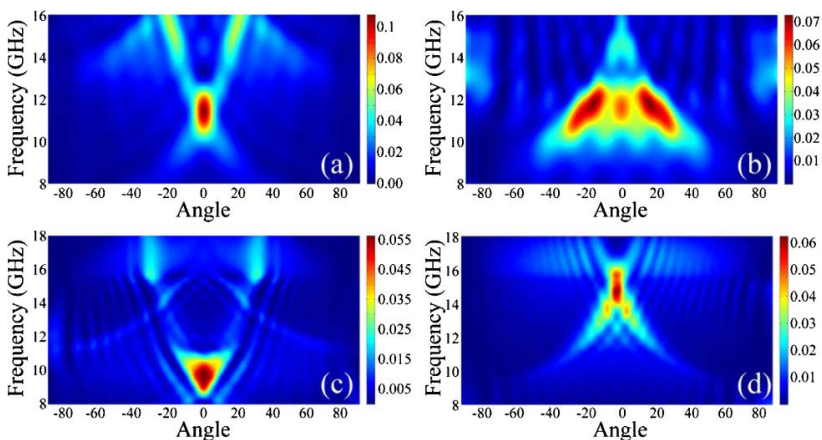

Fig. 2. (Color online) Maps of transmittance for Samples 1 and 2: (a) front-side illumination of Sample 1, (b) back-side illumination of Sample 1 (no tilting), (c) front-side illumination of Sample 2, (d) back-side illumination of Sample 2 (tilting by $30 \mathrm{deg}$ ). Observation angle is measured in the clockwise direction from the normal to the output interface.
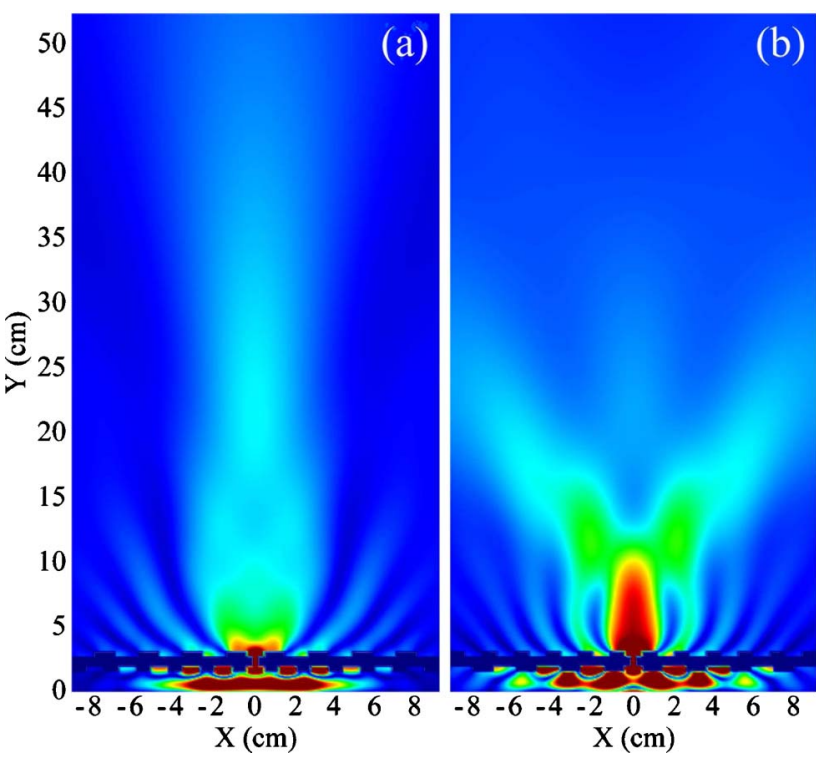

Fig. 3. (Color online) Electric field distribution for Sample 1 at $f=11.2 \mathrm{GHz}$ : (a) front-side and (b) back-side illumination; no tilting. Color bar is normalized to 1 ; maximum is brown.

ing regime and exploits the coupling of surface plasmons to higher diffraction orders.

Electric-field distribution has been calculated at the frequencies corresponding to significant transmission in order to demonstrate the effect of the distance away from the grating. The field distribution at $f=11.2 \mathrm{GHz}$ for Sample 1 is shown in Fig. 3. One can see that it strongly depends on which side is illuminated. The reason is that the period of the output surface determines the grating wavevectors, $k_{g}=2 \pi / \lambda_{g}$, where $\lambda_{g}$ is the grating period, which plays a crucial role in the spatial distribution of the transmitted wave [10]. For the smaller distances, transmission is higher for the back-side illumination. The situation becomes different when moving toward the far zone. For example, the maximal value of transmittance is

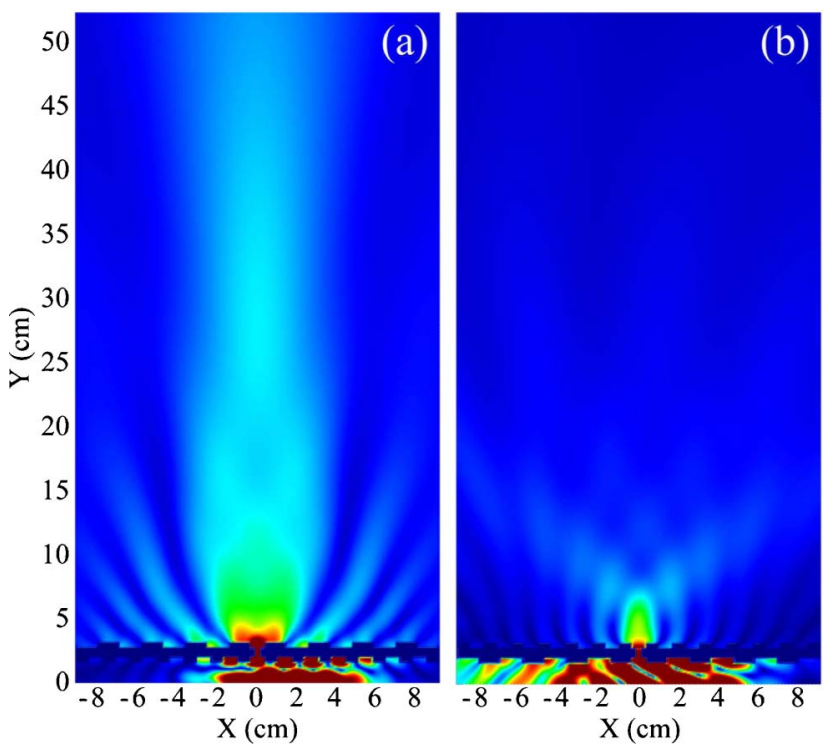

Fig. 4. (Color online) Electric field distribution for Sample 2 at $f=9.8 \mathrm{GHz}$ : (a) front-side and (b) back-side illumination; tilting by $30 \mathrm{deg}$. Color bar is normalized to 0.6 ; maximum is brown. 

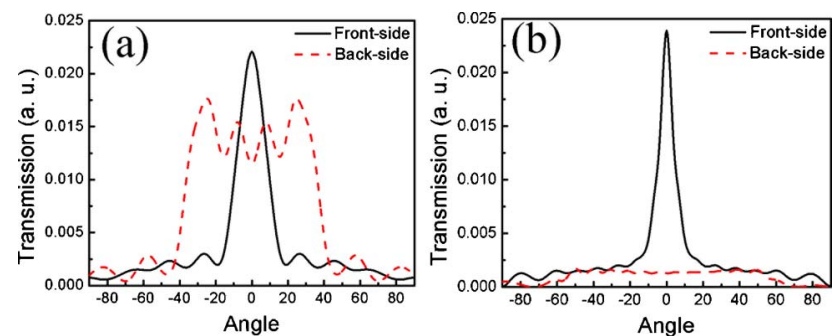

Fig. 5. (Color online) Far-field transmission for (a) Sample 1 at $f=11.2 \mathrm{GHz}$ (without tilting) and (b) Sample 2 at $f=9.8 \mathrm{GHz}$ (tilting by $30 \mathrm{deg}$ ). Solid black curve, front-side illumination; dashed red curve, back-side illumination.

larger in case of the front-side illumination, at least starting from $Y=20 \mathrm{~cm}$. These results are consistent with those in Figs. 2(a) and 2(b).

The calculated electric field distributions for Sample 2 are presented in Figs. 4(a) and 4(b). One can clearly see that the unidirectional transmission can occur within a wide range of the distances from the grating. When Sample 2 is illuminated from the front side at $f=9.8 \mathrm{GHz}$, a much stronger transmission takes place compared to the case where it is illuminated from the back side. Frontside illumination can generate the strong beaming at $9.8 \mathrm{GHz}$. A similar effect can be observed, for example, at $f=14.5 \mathrm{GHz}$; see Fig. 2. However, in this case, a strong transmission is generated by the back-side illumination only. To better understand directional selectivity at large distances, transmission $1 \mathrm{~m}$ away from the center of the structure was calculated. The results are shown in Fig. 5. The only new feature that appears in Fig. 5(a) compared with Figs. 2 and 3 is the large number of local maxima in the case of the back-side illumination. No new feature is observed in Fig. 5(b) compared with Figs. 2 and 4 . Therefore unidirectional beaming is expected to be obtained in the far-field region.

To summarize, we demonstrated that one-way transmission can appear in the beaming regime in nonsymmetric metallic gratings with a subwavelength slit by changing the illumination side. The transmission characteristics can differ strongly, because the output surface is responsible only for the spatial distribution of the transmitted field. It is expected that the suggested mechanism can be implemented for a wide frequency range, including when frequency dispersion of metal cannot be neglected.

This work is supported by the European Union, under the projects EU-PHOME and EU-ECONAM, and TUBITAK under Projects 107A004 and 107A012. E. O. also acknowledges partial support from the Turkish Academy of Sciences. A. S. is supported in part by the Deutsche Forschungsgemeinshaft (DFG) under project SE1409.2-1.

\section{References}

1. Z. Wang, J. D. Chong, J. D. Joannopoulos, and M. Soljacic, Phys. Rev. Lett. 100, 013905 (2008).

2. A. Figotin and I. Vitebskiy, Phys. Rev. B 67, 165210 (2003).

3. M. Scalora, J. R. Dowling, C. M. Bowden, and M. J. Bloemer, J. Appl. Phys. 76, 2023 (1994).

4. M. J. Lockyear, A. P. Hibbins, K. R. White, and J. R. Sambles, Phys. Rev. E 74, 056611 (2006).

5. A. E. Serebryannikov, Phys. Rev. B 80, 155117 (2009).

6. A. E. Serebryannikov and E. Ozbay, Opt. Express 17, 13335 (2009).

7. H. J. Lezec, A. Degiron, E. Devaux, R. A. Linke, L. MartinMoreno, F. J. Garcia-Vidal, and T. W. Ebbesen, Science 297, 820 (2002).

8. H. Caglayan, I. Bulu, and E. Ozbay, J. Opt. Soc. Am. B 23, 419 (2006).

9. S. Kim, H. Kim, Y. Lim, and B. Lee, Appl. Phys. Lett. 90, 051113 (2007).

10. H. Caglayan, I. Bulu, and E. Ozbay, J. Appl. Phys. 104, 073108 (2008).

11. H. Caglayan, I. Bulu, and E. Ozbay, Appl. Phys. Lett. 92, 092114 (2008).

12. H. Raether, Surface Plasmons on Smooth and Rough Surfaces and on Gratings (Springer, 1988).

13. J. B. Pendry, L. Martin-Moreno, and F. J. Garcia-Vidal, Science 305, 847 (2004).

14. P. Hibbins, B. R. Evans, and J. R. Sambles, Science 308, 670 (2005).

15. T. Thio, K. M. Pellerin, R. A. Linke, H. J. Lezec, and T. W. Ebbesen, Opt. Lett. 26, 1972 (2001). 